\title{
Pneumococcal carriage among children under five in Accra, Ghana, five years after the introduction of pneumococcal conjugate vaccine
}

Nicholas T. K. D. Dayie, Elizabeth Y. Tettey', Mercy J. Newman, Elizabeth Bannerman', Eric S. Donkor', Appiah-Korang Labi ${ }^{1}$ and Hans-Christian Slotved ${ }^{2 *}$

\begin{abstract}
Background: The study objective was to determine the carriage and serotype distribution of Streptococcus pneumoniae among children in Accra, Ghana, five years after the introduction of the pneumococcal conjugate vaccine (PCV-13) in 2012.

Methods: Nasopharyngeal swab samples were collected from 410 children below 5 years of age in Accra, Ghana, from September to December, 2016. Pneumococcal isolates were identified by optochin sensitivity and bile solubility. Serotyping was performed using the latex agglutination kit and Quellung reaction. The isolates were furthermore tested for antimicrobial susceptibility for different antimicrobials, including penicillin (PEN). Twelve isolates including seven non-typeable (NT) isolates were characterized using whole-genome sequencing analysis (WGS).

Results: The overall carriage prevalence was found to be $54 \%$ (95\% Cl, 49-59\%), and 20\% (95\% Cl, 49-59\%) of the children were carrying PCV-13 included serotypes, while $37 \%(95 \% \mathrm{Cl}, 33-42 \%)$ of the children were carrying nonPCV-13 serotypes. Based on the serotype distribution, 33\% of all observed serotypes were included in PCV-13 while $66 \%$ were non-PCV-13 serotypes. The dominating non-PCV-13 serotypes were $23 \mathrm{~B}, 16 \mathrm{~F}$, and $11 \mathrm{~A}$ followed by PCV13 serotypes $23 \mathrm{~F}$ and $19 \mathrm{~F}$. The PCV-13 covers the majority of resistant isolates in Accra. A proportion of $22.3 \%$ of the isolates showed intermediate resistance to penicillin $\mathrm{G}$, while only one isolate showed full resistance. Forty-five isolates (20.5\%) were defined as multidrug-resistant (MDR) as they were intermediate/resistant to three or more classes of antimicrobials. Of the seven NT isolates characterized by WGS, four showed highest match to genotype 38, while the remaining three showed highest match to genotype 14. Four MDR serotype 19A isolates were found to be MLST 320.

Conclusion: PCV-13 introduced in Ghana did not eliminate PCV-13 covered serotypes, and the carriage rate of 54\% in this study is similar to carriage studies from pre PCV-13 period. However, the penicillin non-susceptible isolates have been reduced from $45 \%$ of carriage isolates before PCV-13 introduction to $22.3 \%$ of the isolates in this study. Continuous monitoring of serotype distribution is important, and in addition, an evaluation of an alternative vaccination schedule from $3+0$ to $2+1$ will be important to consider.
\end{abstract}

Keywords: Streptococcus pneumoniae, Ghana, Carriage, Serotype, PCV-13

\footnotetext{
* Correspondence: hcs@ssi.dk

${ }^{2}$ Department of Bacteria, Parasites and Fungi, Statens Serum Institut,

Artillerivej 5, DK-2300 Copenhagen, Denmark

Full list of author information is available at the end of the article
}

(c) The Author(s). 2019 Open Access This article is distributed under the terms of the Creative Commons Attribution 4.0 International License (http://creativecommons.org/licenses/by/4.0/), which permits unrestricted use, distribution, and reproduction in any medium, provided you give appropriate credit to the original author(s) and the source, provide a link to the Creative Commons license, and indicate if changes were made. The Creative Commons Public Domain Dedication waiver (http://creativecommons.org/publicdomain/zero/1.0/) applies to the data made available in this article, unless otherwise stated. 


\section{Background}

Streptococcus pneumoniae (pneumococcus) is considered the leading pathogen associated with community-acquired pneumonia, otitis media and meningitis [1]. Pneumococcal appearance in humans can be divided into two phases, carriage and the disease phase, where the carriage of $S$. pneumoniae is generally described as the prerequisite for developing pneumococcal infections, and often young children are considered to act as reservoirs [2, 3]. Pneumococcal infections have attracted global public health attention due to the high burden of disease and associated mortality, particularly among children under five and adults $>64$ years in resource poor countries $[1,4,5]$. The high burden of morbidity and mortality associated with the pneumococcus can be reduced using appropriate vaccines. Hence, recently attention has been given to the introduction of pneumococcal conjugate vaccines into the children vaccination programmes in the developed and developing countries $[1,5,6]$.

Since 2000, studies have shown that the introduction of the pneumococcal conjugate vaccines (PCV7, PCV-10 and PCV-13 in selected countries) has been effective particularly among children under 5 years [1,7-10]. Based on these results, the vaccines have been introduced in other parts of the world including Africa [5, 6, 11].

However, to be able to measure the impact of PCVs, it is important to have pre-vaccination data on the serotype distribution [10-12]. Several studies from Africa prior to the introduction of PCVs have been performed and showed that the major serotypes were $1,5,6 \mathrm{~A}, 6 \mathrm{~B}$, $14,19 \mathrm{~A}, 19 \mathrm{~F}$ and $23 \mathrm{~F}[4,10,11,13]$. In Ghana, the PCV13 was introduced as part of the routine childhood immunization programme in May 2012, using the $3+0$ vaccination schedule $[6,13]$. The official country report on PCV13 coverage was estimated to be $99 \%$ in 2017 (http://www.view-hub.org, accessed 02-09-2019). Prior to the PCV-13 introduction in Ghana, several studies showed the nature and distribution of pneumococcal serotypes circulating in Ghana [13-16]. The carriage study by Dayie et al. [13] showed that the predominant serotypes were $19 \mathrm{~F}, 6 \mathrm{~B}, 23 \mathrm{~F}$ and $6 \mathrm{~A}$, and a $\mathrm{PCV}-13$ vaccine coverage was estimated to be approximately $50 \%$. Other studies have shown that the introduction of the PCV in the routine childhood immunization programme has reduced the carriage of vaccine serotypes but there has been an increase in non-PCV serotypes $[2,10]$. Studies from the Gambia showed that PCV-7 and PCV-13 had a positive effect on the vaccine-type carriage (VT-carriage), while an increase in the carriage prevalence of non-PCV serotypes was observed $[5,10]$. Five years after the introduction of the pneumococcal conjugate vaccine in Ghana, there is no post PCV-13 data on prevailing circulating serotypes to measure the impact of PCV-13 among the healthy Ghanaian population. In addition, the previous study by Dayie et al. [17] showed an increasing incidence of multidrug resistant pneumococci among carriage isolates; hence, data to determine the impact of $\mathrm{PCV}-13$ vaccinations and the trend of antibiotic resistance in pneumococci among children under five is needed.

The aim of this study was to determine the pneumococcal serotype distribution and antimicrobial susceptibility patterns of carriage isolates among healthy children ( $\leq 5$ years), five years post PCV-13 vaccination in Accra, Ghana,.

\section{Methods}

Study sites

The study was carried out in the Accra metropolis, which is the capital city of Ghana and falls within the coastal belt with humid and warm climatic conditions. Accra has the second highest population density compared to other districts in Ghana (https://www.indexmundi.com/ghana/demographics_profile.html;

accessed 02-09-2019). The PCV-13 is part of the routine childhood immunization programme in Ghana, and the vaccination schedule is 6,10 and 14 weeks [13].

\section{Sampling and study design}

The study was carried out in nurseries and kindergartens within the Accra metropolis of the Greater Accra region of Ghana from September to December 2016.

A list of nurseries and kindergartens in the Accra metropolis was obtained from the Ghana Education service. Seven schools were randomly selected and written consent was obtained from the parents of the children. Children whose parents declined to give their consents were excluded from the study; children who declined assent after parental consent were also excluded. Children with active upper respiratory tract infections or who had been given antibiotics within the last two weeks prior to sampling were excluded. Postnatal cards were obtained from the parents in order to ascertain the vaccination status of the children.

\section{Specimen collection}

Nasopharyngeal specimens were collected using a WHO recommended methodology [16]. From September to December 2016, nylon-tipped paediatric sized FlOQSwabs (Copan Flock Technologies, Italy) were used to collect nasopharyngeal specimens. Four hundred and ten swab samples were obtained. Immediately after collection the swab specimens were placed in premade vials containing $1 \mathrm{ml}$ of skim milk-tryptone-glucose-glycerin (STGG) medium and transported on ice to the laboratory within $3 \mathrm{~h}$ of collection. Upon arrival at the laboratory the swab samples were immediately stored at $-80^{\circ} \mathrm{C}$ pending further processing [18]. 


\section{Characterization of S. pneumoniae}

The specimens were processed based on the WHO recommendation for characterizing S. pneumoniae [18]. The samples were inoculated onto a 5\% sheep blood agar containing $5 \mu \mathrm{g} / \mathrm{ml}$ of Gentamicin and then incubated at $37^{\circ} \mathrm{C}$ in $5 \% \mathrm{CO}_{2}$ for $18-24 \mathrm{~h}$. A representative number of alpha-haemolytic colonies were subjected to optochin susceptibility testing, and based on the visual evaluation and the isolates' susceptibility to optochin (inhibition zone $\geq 14$ $\mathrm{mm}$ ), swab samples were identified as containing possible S. pneumoniae. All swab samples suspected to contain $S$. pneumoniae isolates were transported on dry ice to Statens Serum Institut (SSI), Copenhagen, Denmark for further characterization. At SSI, the organisms were isolated from the swab samples and verified as pneumococcal isolates using phenotypic methods as described in previous studies $[2,19]$. Briefly, $10 \mu \mathrm{l}$ of the swab samples were cultured in serum broth overnight, the following day $1 \mu \mathrm{l}$ of each serum broth was cultured on $10 \%$ horse blood agar plates and incubated overnight at $37^{\circ} \mathrm{C}, 5 \% \mathrm{CO} 2$. All serum broths were screened for multiple serotypes by using the Pneumotestlatex agglutination kit (SSIDiagnostica, Denmark) [2]. Serotyping/grouping of the isolates was performed using the Pneumotest-latex agglutination kit (SSIDiagnostica, Denmark) and the results were confirmed by the Quellung reaction test using the serotype specific antisera (SSIDiagnostica, Denmark) [19]. Non-typeable strains were defined as isolates presenting no phenotypic detectable capsule.

\section{Characterization of selected isolates}

Due to financial constraints, we were only able to perform whole genome sequencing (WGS) on seven of the ten NT isolates and five of the multidrug-resistant (MDR) isolates. WGS was performed on 12 isolates. The isolates were sequenced by paired-end Illumina sequencing. Genomic DNA was extracted using a DNeasy Blood \& Tissue Kit (QIAGEN, Hilden, Germany) and fragment libraries were constructed using a Nextera XT Kit (Illumina, Little Chesterford, UK) followed by 250bp paired-end sequencing (MiSeqTM; Illumina) according to the manufacturer's instructions. The paired-end Illumina data were de novo assembled using CLCbio's Genomics Workbench v.7.5 QIAGEN) reporting only contigs $>500$ bp using standard settings.

Bioinformatics, including blast, was done using the software CLC Main Workbench (Version 7.9.1, www. qiagenbioinformatics.com).

Multilocus Sequence Analysis (MLSA) as described by Bishop et al. [20], and the presence of cytosine at the 203 position using the 16S rRNA sequence [21] confirmed the pneumococcal species identification for all 12 isolates. The presence/absence of a gene was based on a cut-off of $80 \%$ coverage and a $95 \%$ identity for positive gene detection in this study [22].
The presence of capsular genes for all 12 isolates were blasted for 92 capsular polysaccharide genes (CPS genes) as described by Kapatai et al [22].

Multilocus sequence typing (MLST) was performed using the PubMLST DataBase (https://pubmlst.org/ spneumoniae/) to identify the sequence type (ST) for each of the isolates.

The isolates were also analyzed for their PenicillinBinding Protein (PBP) signature, based on a genotyping proposal and algorithm described for PBP1A, PBP2B and PBP2X [23], where the combination of the three PBP signatures determines the level of beta-lactam resistance. The isolates were tested by blast with the published types of predictive mutations vs. resistance levels of PBP1A, PBP2B and PBP2X proteins as described in $\mathrm{Li}$ et al. [23] and CDC (https://www.cdc.gov/streplab/ pneumococcus/mic.html, accessed 18-06-2019).

Also, the presence of the genes ermB and tet were tested, and ResFinder 3.0 (https://cge.cbs.dtu.dk/services/ResFinder/) (80\% ID threshold and 60\% minimum length settings) was used to confirm the presence of the three genes [24].

\section{Antimicrobial susceptibility testing}

Penicillin susceptibility testing was initially determined by agar-disc diffusion using $1 \mu \mathrm{g}$ oxacillin disc (Oxoid Company, UK). Minimum inhibitory concentrations (MICs) for all oxacillin resistant isolates $(\mathrm{R}<20)$ were determined using penicillin G MIC strips (Oxoid Company, UK).

Penicillin (PEN) susceptibility was defined as susceptible $(\mathrm{MIC} \leq 0.06 \mu \mathrm{g} / \mathrm{ml})$, intermediate $(>0.06-2 \mu \mathrm{g} / \mathrm{ml})$ and resistant $(>2 \mu \mathrm{g} / \mathrm{ml})$ according to the European Committee on Antimicrobial Susceptibility Testing (EUCAST) guidelines with S. pneumoniae ATCC 49619 used as a control (EUCAST Clinical Breakpoint Tables v. 6.0, valid from 2016 to $01-01$ ).

All isolates were further tested using the disc diffusion method against erythromycin (ERY) $(15 \mu \mathrm{g}$ disk), tetracycline (TET) $(30 \mu \mathrm{g}$ disk), trimethoprim-sulphamethoxazole (SXT) (1.25/23.75 $\mu \mathrm{g}$ disk) and levofloxacin (LEV) (5 $\mu \mathrm{g}$ disk). The susceptibility test using Oxoid disks (Oxoid Company, UK) was performed by spreading an inoculum of $0.5 \mathrm{McF}$ arland standard onto Müller-Hinton (Oxoid, UK) agar plates containing $5 \%$ sheep blood. The plates were incubated between 18 and $24 \mathrm{~h}$ at $37^{\circ} \mathrm{C}$ in a $5 \% \mathrm{CO}_{2}$ incubator, after which the zones of inhibition were measured with a calliper.

Multidrug-resistant (MDR) isolates are defined as isolates showing resistance (intermediate or resistant) to at least three classes of antimicrobials [9].

\section{Data analysis}

Data were analyzed using Graph Pad Prism version 7 (GraphPad Software) for descriptive statistical analysis. R version 3.5.0 (2018-04-23) was used for calculation of 
confidence intervals (95\% CI) and for the logistic regression model using the glm function in $\mathrm{R}(\mathrm{R}$ version 3.5.0 (2018-04-23) for calculations in the univariable and multivariable model. $P$-value $<0.05$ was considered significant.

\section{Results}

\section{Characteristics of the study group}

Four hundred and ten children participated in the study with almost an equal distribution of gender $(52.5 \%$ were male). The mean age of the group was 39 months with a range of 6 months to 60 months of age. Four hundred and seven (407) of the 410 children were fully vaccinated with three doses of PCV-13 vaccines, two were of unknown vaccination status; one child was unvaccinated. All children with detected carriage were vaccinated with three doses of PCV-13 (Table 1).

\section{Carriage rate and serotype distribution}

The observed overall carriage rate was 54\% (95\% CI, 49-59\%) with nearly identical carriage rate between male and female children (Table 1). Because there was no difference in carriage by sex, only the OR and not adjusted OR was calculated for each age group (Table 1). The age group 36-47 month showed the highest carriage rate of 59\% (95\% CI, 52-67\%) (Table $1)$. Two hundred and thirty four pneumococcal isolates were isolated from 220 children of which 14 children harbored two different pneumococcal serotypes (Table 1).

The predominant serotypes observed were the nonvaccine serotypes $23 \mathrm{~B}(11 \%)$ and $16 \mathrm{~F}(10 \%)$ followed by the vaccine serotypes $23 \mathrm{~F}(8 \%)$ and $19 \mathrm{~F}(6 \%)$. The dominating PCV-13 serotypes were 23F, 19F, 19A (6\%) while non-PCV-13 serotypes were 23B, 16F, 11A (7\%), and 34

Table 1 Characteristics of participating children

\begin{tabular}{|c|c|c|c|c|}
\hline & $\begin{array}{l}\text { Total number of } \\
\text { children }\end{array}$ & $\begin{array}{l}\text { Number of children with carriage of } S \text {. } \\
\text { pneumoniae }(\%, 95 \mathrm{Cl})\end{array}$ & $\begin{array}{l}\mathrm{OR}(95 \% \mathrm{Cl})^{*} \\
(p \text {-value })^{*}\end{array}$ & \\
\hline Overall carriage rate & 410 & $220(54,49-59)$ & & \\
\hline Females carriage rate & 200 & $108(54,47-61)$ & 1 & \\
\hline Males carriage rate & 210 & $112(53,47-60)$ & $\begin{array}{l}0.97(0.66-1.44) \\
(P=0.892)\end{array}$ & \\
\hline $\begin{array}{l}\text { Number of Children carrying } \\
\text { PCV-13 serotypes }\end{array}$ & 410 & $81(20,16-24)$ & & \\
\hline \multirow{2}{*}{$\begin{array}{l}\text { Number of Children carrying } \\
\text { non-PCV-13 serotypes }\end{array}$} & 410 & $153(37,33-42)$ & & \\
\hline & Median age (Month) & Range (Month) & $\begin{array}{l}\text { Interquartile } \\
\text { range (month) }\end{array}$ & \\
\hline Children & 36 & $(6-60)$ & $36-48$ & \\
\hline Males & 36 & $(6-60)$ & $36-48$ & \\
\hline \multirow[t]{2}{*}{ Females } & 36 & $(12-60)$ & $36-48$ & \\
\hline & $\begin{array}{l}\text { Vaccination status of } \\
\text { all participants }\end{array}$ & Vaccination status of carriers & & \\
\hline PCV13 vaccinated & 407 (99.3\%) & $220(100 \%)$ & & \\
\hline Unknown & 2 & 0 & & \\
\hline Not vaccinated & 1 & 0 & & \\
\hline Age group (months) & $\begin{array}{l}\text { Total number of } \\
\text { children }\end{array}$ & $\begin{array}{l}\text { Number of children with carriage of } \mathrm{S} \text {. } \\
\text { pneumoniae }(\%)(95 \% \mathrm{Cl})\end{array}$ & $\begin{array}{l}\text { OR }(95 \% \mathrm{Cl})^{*} \\
(p \text {-value })^{*}\end{array}$ & $\begin{array}{l}\text { Number of children with } \\
\text { multiple serotypes }\end{array}$ \\
\hline $0-11$ & 2 & $1(50,13-200)$ & 1 & 0 \\
\hline $12-23$ & 22 & $9(40,25-68)$ & $\begin{array}{l}0.69(0.03-19.06) \\
(P=0.804)\end{array}$ & 0 \\
\hline $24-35$ & 73 & $41(56,46-69 \%$ & $\begin{array}{l}1.28(0.05-33.24) \\
(P=0.863)\end{array}$ & 3 \\
\hline $36-47$ & 178 & $105(59,52-67 \%)$ & $\begin{array}{l}1.44(0.05-36.76) \\
(P=0.798)\end{array}$ & 10 \\
\hline $48-60$ & 135 & $64(47)(40-57 \%)$ & $\begin{array}{l}0.90(0.03-23.11) \\
(P=0.942)\end{array}$ & 1 \\
\hline Total & 410 & 220 (54) (49-59\%) & & 14 \\
\hline
\end{tabular}

*Odds ratios, confidence intervals and $p$-values were calculated using a generalized linear model (glm function in $\mathrm{R}$ ) a. Because 14 children were carrying two different serotypes, the number of children carrying the multiple serotupes in these two groups exceed 220 children and the figure is currently 234 cases 
(6\%) (Fig. 1). Ten isolates were found to be non-typeable isolates (Table 1).

$34.6 \%$ of the detected serotypes were covered by the PCV-13, and $65.4 \%$ of the isolates (including NT isolates) were found to be non-PCV-13 serotypes (Table 2).

In Additional file 1: Table S1, the serotype distribution data from Dayie et al. [2013] and the data from the present study has been presented, thereby making it possible to compare the data set from the two carriage studies.

\section{Antimicrobial resistance}

$22.3 \%$ of the isolates showed intermediate resistance to penicillin G, while one isolate showed penicillin resistance (Table 2).

The highest number of resistant isolates was observed for tetracycline (63\%) and trimethoprim-sulphamethoxazole $(61.4 \%)$ of which more than half of the strains showed resistance. In addition, two isolates were tetracycline intermediate resistant while $14.5 \%$ were trimethoprim-sulphamethoxazole intermediate resistant. It was observed that $11 \%$ of the isolates were resistant to erythromycin while $5 \%$ of the isolates were intermediate resistant (Table 2, Fig. 2). All isolates were sensitive to levofloxacin. $65.5 \%$ (144 isolates) of intermediate/resistant isolates were serotypes not included in the PCV-13 (Table 2).

Twenty-seven isolates $(12.3 \%)$ were found to be intermediate/resistant to three classes of antimicrobials, while 18 isolates $(8.2 \%)$ were intermediate/resistant to four classes of antimicrobials (Fig. 2). In total 45 isolates (20.5\%) were defined as MDR isolates as they were intermediate/resistant to three or more classes of antimicrobials. Twenty-eight isolates of the $45 \mathrm{MDR}$ isolates were covered by the PCV-13 vaccine (Fig. 2).

\section{Molecular characteristics of 12 isolates}

Four of the seven analyzed NT isolates showed a preference for genotype 38 showing the highest hit score (Table 3). Three of these isolates (G10, C131 and C28) showed identical MLST presenting a novel ST within the Clonal Complex ST908 (the seven loci $d d l$ was unidentified) (Table 3). The fourth isolate (G11) was ST344, and was completely different from the other three isolates. The remaining three NT isolates showed the highest hit score for genotype 14 (Table 3). Two of the isolates G7 with ST (2-14-37-36-29-17-21) and G140 with ST9735 were relatively closely related with only one locus difference (the recP locus). The third isolate (C139) was not related to the two other isolates (G7 and G140).

With the five tested MDR isolates, the serotypes were confirmed by the genotypes and STs (Table 3).

Four serotype 19A isolates (G14, G27, G28, C88) were found to be MDR isolates. All four isolates were ST 320 and showed the same PBP profile $(13,11,16)$. The PBP profile corresponded to the phenotypic susceptibility profile as penicillin intermediate (Table 2). A fifth serotype 15 MDR isolate (D012) also showed a PBP profile corresponding to the phenotypic susceptibility profile.

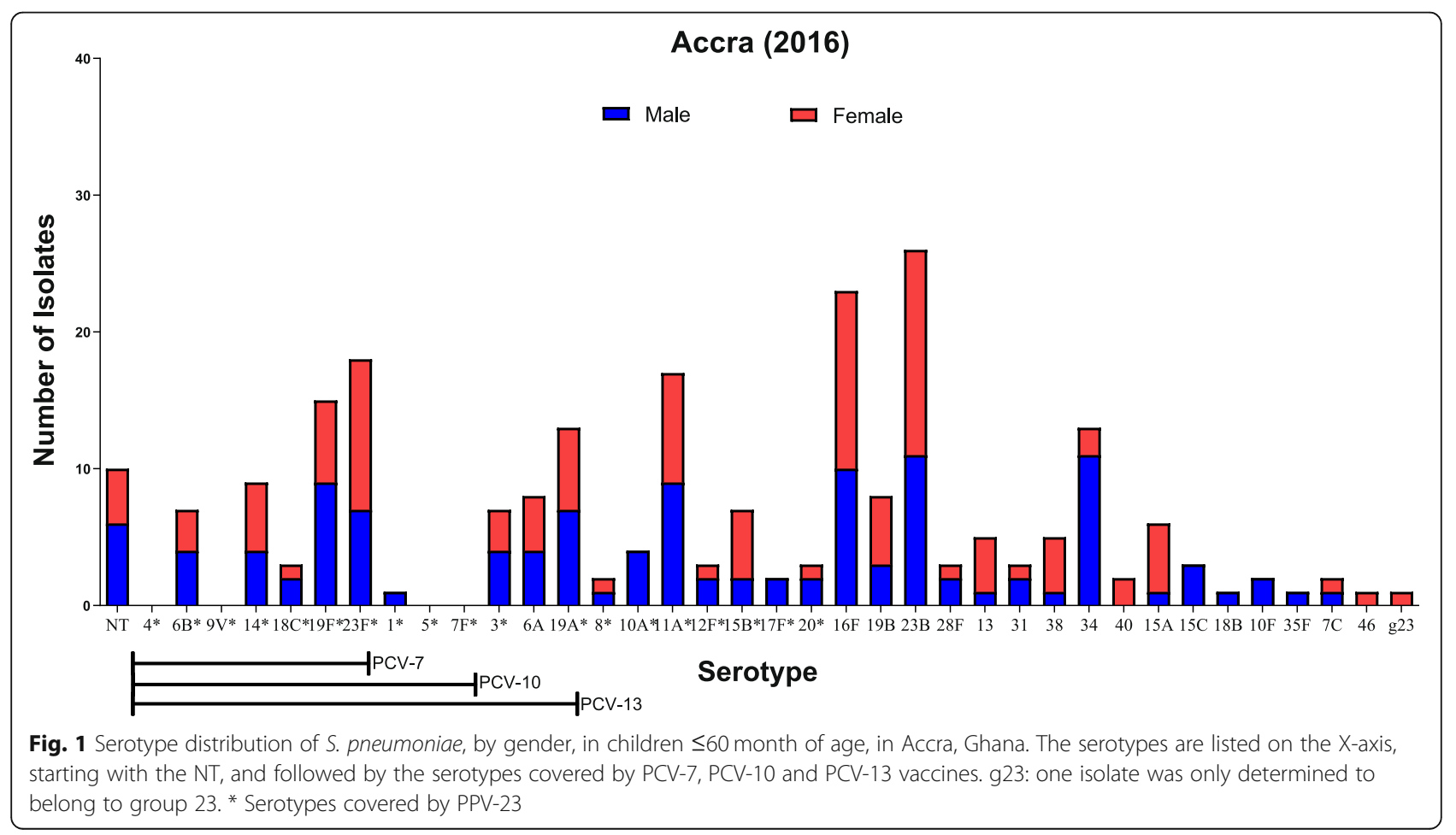


Table 2 Distribution of S. pneumoniae isolates with intermediate/full resistance towards four antimicrobials by vaccine coverage. All isolates were sensitive to Levofloxacin

\begin{tabular}{llllll}
\hline & $\begin{array}{l}\text { Number of isolates } \\
\text { tested for } \\
\text { susceptibility }\end{array}$ & $\begin{array}{l}\text { Number of non-susceptible } \\
\text { isolates (\% of all isolates) }\end{array}$ & $\begin{array}{l}\text { Number of } \\
\text { non-typable } \\
\text { tested for } \\
\text { susceptibility }\end{array}$ & $\begin{array}{l}\text { Number of } \\
\text { non-susceptible } \\
\text { PCV-13 serotypes }\end{array}$ & $\begin{array}{l}\text { Number of } \\
\text { non-susceptible } \\
\text { non-PCV13 serotypes }\end{array}$ \\
\hline $\begin{array}{llll}\text { All isolates } \\
\text { PEN (MIC 0.06-2) }\end{array}$ & 220 & $191(86.8 \%)$ & $10(4.5 \%)$ & $76(34.5 \%)$ & $144(65.5 \%)$ \\
PEN (MIC $>$ 2) & $220^{\text {a }}$ & $49(22.3 \%)$ & $7(3.2 \%)$ & $27(12.3)$ & $13(6.0 \%)$ \\
TET $(25>1>=22)$ & $219^{b}$ & $1(0.5 \%)$ & 0 & 0 & $1(0.5 \%)$ \\
TET $(R<22)$ & & $2(0.9 \%)$ & 0 & $1(0.5 \%)$ & $1(0.5 \%)$ \\
SXT $(18 \geq 1 \geq 15)$ & $220^{c}$ & $138(63.0 \%)$ & $7(3.2 \%)$ & $64(29.2 \%)$ & $51(2.3 \%)$ \\
SXT $(R<15)$ & & $32(14.5 \%)$ & $8(3.6 \%)$ & $10(4.5 \%)$ & $18(8.2 \%)$ \\
ERY $(S \geq 22>1 \geq 19)$ & $220^{d}$ & $135(61.4 \%)$ & $1(0.5 \%$ & $4(1.8 \%)$ & $43(19.5 \%)$ \\
ERY $(R<19)$ & & $11(5.0 \%)$ & 0 & $15(6.8 \%)$ & $5((2.3 \%)$ \\
MDR $\geq 3$ & 220 & $24(10.9 \%)$ & $4(1.8 \%)$ & $27(12.3)$ & $8(3.6 \%)$ \\
\hline
\end{tabular}

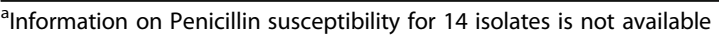

${ }^{b}$ Information on Tetracycline susceptibility for 15 isolates is not available

Information on SXT susceptibility for 14 isolates is not available

${ }^{d}$ Information on Erythromycin susceptibility for 14 isolates is not available

Eleven isolates harbored the tet $(\mathrm{M})$ gene and four isolates harbored the $\mathrm{erm} B$ gene according to ResFinder 3.0 (Table 3). The presence of ermB gene was generally in agreement with the phenotypic antibiotic susceptibility result, while two of the isolates $(\mathrm{C} 131, \mathrm{C} 28)$ harboring the $\operatorname{tet}(\mathrm{M})$ gene, were still found to be phenotypically sensitive (Table 3). Based on the information from the resistance gene, none of the isolates harboring the ermB gene were found positive for the presence of mobile genetic elements of Tn-family, while Tn917 was found in six isolates harboring the tet $(\mathrm{M})$ gene. The two isolates harboring the tet $(\mathrm{M})$ gene, but still found phenotypical sensitive also showed presence of the transposon Tn916 (Table 3).

\section{Discussion}

Worldwide pneumococcal carriage studies have been performed to measure the impact of the PCV vaccination among children $[5,10,25,26]$. The majority of carriage studies performed in Africa were baseline studies with the purpose of evaluating the effectiveness of the PCVs [5, 12, 25-27]. This study is to our knowledge the first carriage study performed in Accra, Ghana, among healthy children to evaluate the effect of PCV13 on pneumococcal carriage five years after the PCV13 introduction [13]. Only few other studies in the region have performed post $\mathrm{PCV}$ introduction carriage studies $[5,10]$.

The overall carriage rate observed in this study after five years of PCV-13 vaccination was 54\% (95\% CI, 49 59) (Table 1). Two pre-PCV-13 carriage studies from Accra performed in 2011, both on healthy children below 5 years of age, showed a carriage rate of $34 \%$ in nursery/kindergarten children [13] and a 49\% carriage rate in children from a pediatric hospital in Accra [16]. Comparing the carriage rate for PCV-13 serotypes (18\%) and the non-PCV-13 serotypes (19\%) from the pre-PCV vaccination period from Accra [13] with the carriage rate observed in this study for PCV13 serotypes (20,95\% CI, 16-24) and the carriage rate for non-PCV serotypes (37, 95\% CI 33-42) in Accra, show that the PCV13 carriage rate have not changed or increased, while an increase in the non-PCV serotypes carriage rate was observed. The PCV-13 introduction does therefore not seem to have had a reducing effect on the overall carriage rate in children in Accra, Ghana. Other studies have also observed no net effect of the carriage rate after PCV introduction [27]. In the Gambia, they observed no net effect on the carriage rate after 2 years of PCV-7 vaccination [5] and after five years of PCV-13 vaccination [10]. A Danish study also observed that the overall carriage rate in children was not reduced after more than 10 years of PCV vaccination [2].

Before the introduction of the pneumococcal vaccination in Ghana, the PCV-13 showed a coverage of $48 \%$ of the detected carried serotypes [13], while in this study the coverage rate of the PCV-13 was 35\%. The introduction of the PCV-13 in Ghana has had an effect on the serotype distribution although both the overall carriage rate has not been reduced and PCV-13 serotype carriage rate are still the same (between 18 and 20\%) [13]. VTserotypes are, however, still found as carriage serotypes in the vaccinated children in this study. This has also been observed in the study from the Gambia [10] while it was not the case in a Danish carriage study, where VT serotypes rarely were detected [2]. A possible 

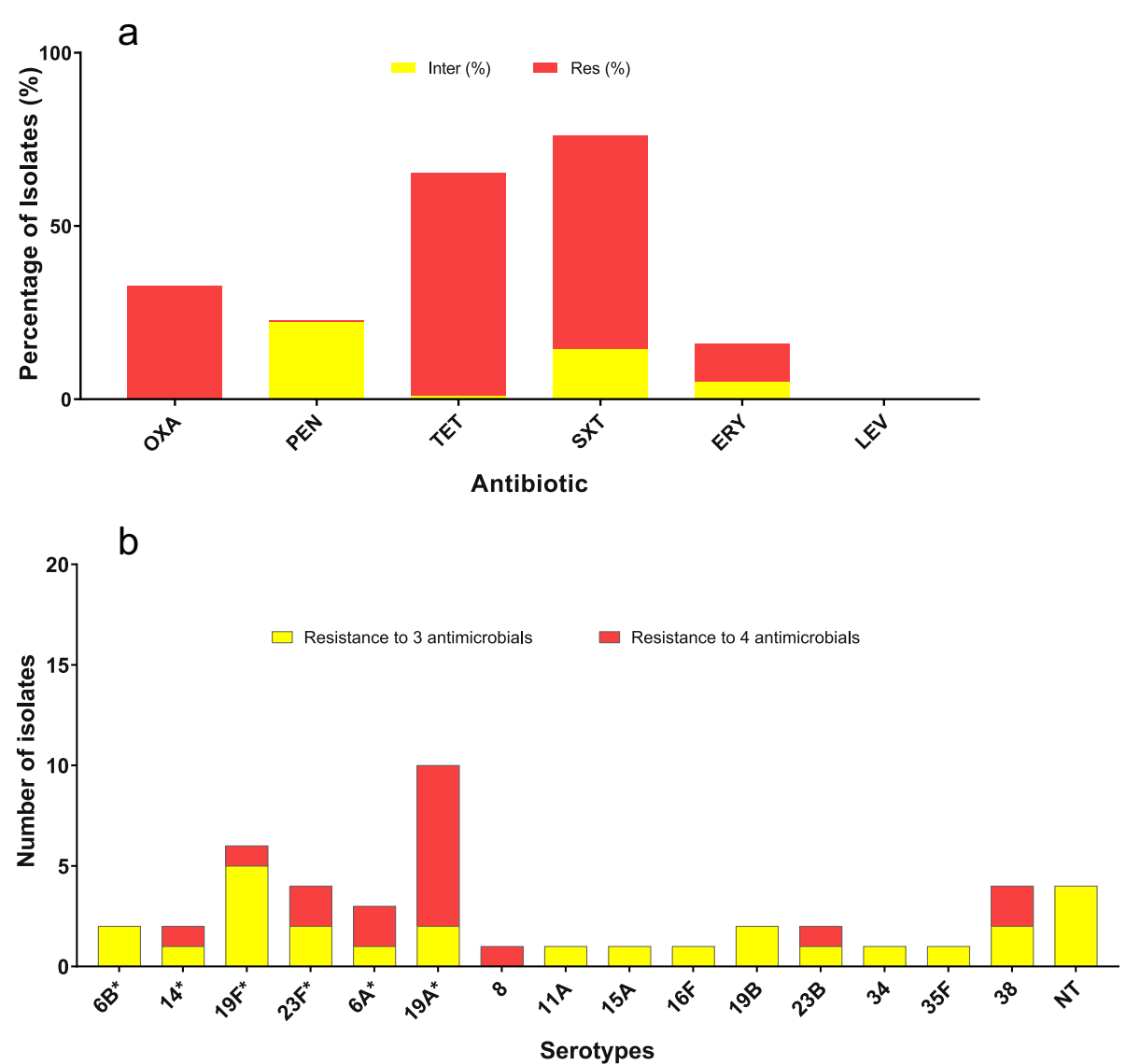

Fig. 2 The antimicrobial susceptibility and serotype distribution of Multidrug-resistant (MDR) defined isolates. MDR isolates are defined as isolates showing resistance (intermediate or resistant) to at least three classes of antimicrobials. Figure 2a presents the MDR $\geq 3$, and Fig. $2 b$ presents the total resistant isolates. Information on Penicillin susceptibility for 14 isolates is not available. ${ }^{b}$ Information on Tetracycline susceptibility for 15 isolates is not available. Information on Trimethoprim-sulphamethoxazole susceptibility for 14 isolates is not available. 'Information on Erythromycin susceptibility for 14 isolates is not available

explanation of this difference might be the vaccination schedule [10], which in the Gambia and Ghana is $3+0$, while it is $2+1$ in Denmark $[2,10]$.

Comparison of the serotype distribution observed in this post PCV-13 study in Ghana with the pre PCV-13 study in 2011 [13] showed that some of the VT-serotypes are still dominating, such as serotypes (6B, 14, 19F and 23F). However, changes in the serotype distribution have been observed, as the carriage prevalence of serotypes $6 \mathrm{~B}$ and 19F in 2011 were 10 and 15\%, respectively, of the detected isolates in Accra, while in 2017, the carriage prevalence of serotypes $6 \mathrm{~B}$ and $19 \mathrm{~F}$ were 3 and $6 \%$, respectively. An increase in carriage prevalence was observed for serotype 19A from about $1.3 \%$ in 2011 to $5.6 \%$ in 2017 . Generally, none of the PCV-13 serotypes in 2017 was found to be more than $8 \%$ of the total serotypes observed.

The overall predominant serotypes in 2017 were serotype 23B and serotype 16F, which are not included in either PCV-13 or PPV-23. In addition, serotype 11A (not included in PCV-13) and serotype 34 (not included in
PCV-13 and PPV-23) were common serotypes. Of the four non-PCV serotypes, only $11 \mathrm{~A}$ is included in the PPV-23, while the three other serotypes are not part of PPV-23 [13]. Although carriage studies cannot provide information on which new replacement serotypes might be the future dominant cause of pneumococcal disease, it can indicate whether the vaccine coverage might continue to be low with regard to detected serotypes [28], and a new PCV vaccine may have limited impact on the pneumococcal epidemiology in Ghana.

The carriage prevalence that was observed between age groups in this study (Table 1) was very similar to both the pre-PCV-studies, where the carriage rates peaked around age groups 24-35 months and 36-47 months [13] and around 43-48 months [16]. Because this study included only a limited number of children younger than 1 year, we cannot describe the effect of the PCV-13 vaccination carriage in this age group. However, other studies in Africa have shown that there is a high carriage also within this age group [5, 10,29]. 


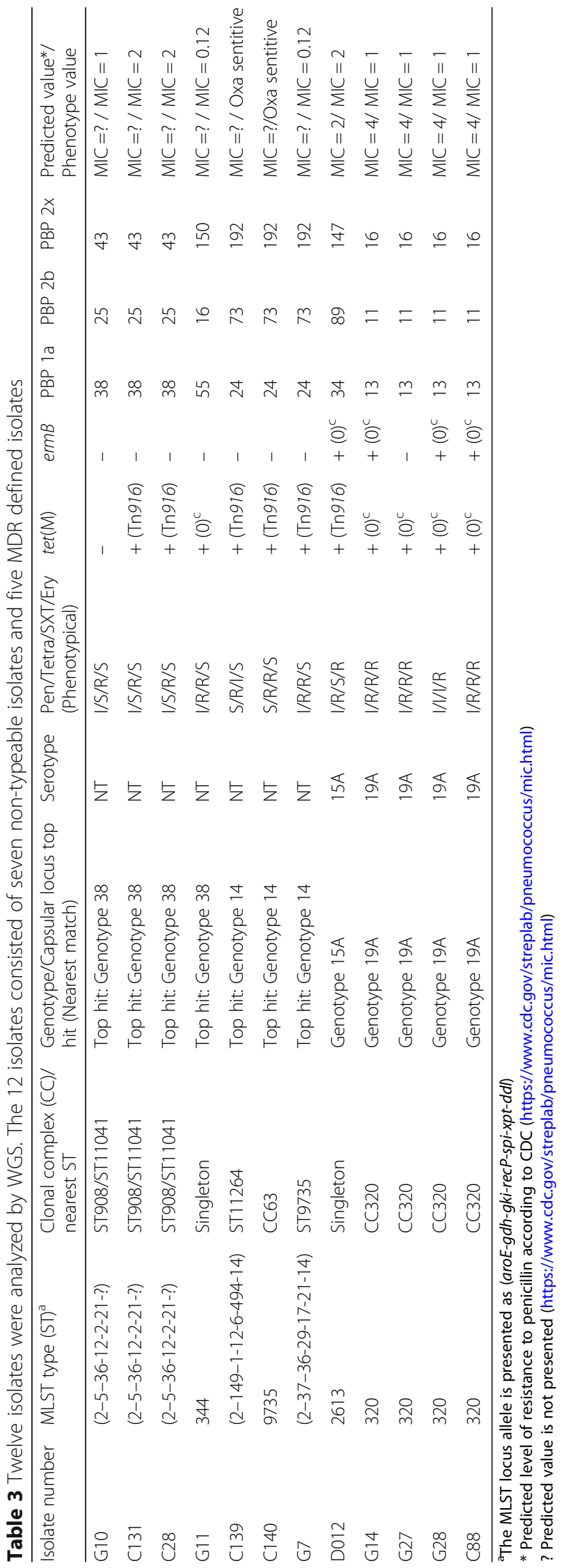


In the pre-PCV-carriage study from Ghana by Dayie et al. [13], 45\% of the isolates from Accra showed penicillin intermediate resistance. Another carriage study from Ghana performed in 2011 also showed a high percentage of penicillin resistance of $63 \%$ [16]. In this study, we observed a decline in the prevalence of penicillin intermediate resistant isolates to $22 \%$ (Table 2).

In Ghana, the Standard Treatment Guidelines (Sixth Edition, 2010 - Ghana) recommend clinicians generally to use amoxiclav or the penicillin for pediatric infections (http://apps.who.int/medicinedocs/en/m/abstract/Js1

$8015 \mathrm{en} /$, accessed 02-09-2019). The decline in prevalence of penicillin intermediate resistance in this study may be attributed to the effect of PCV-13 vaccination, which was shown to cover more than half of the intermediate penicillin resistant isolates observed in the study by Dayie et al. [13].

While the PCV-13 vaccination seems to have reduced penicillin resistance in Ghana, this does not appear to be the case with tetracycline, which this study found to be about $63 \%$, while previous pre-PCV studies have shown similar or higher tetracycline resistance of about $60-85 \%$ $[16,30]$. In a carriage study in 2007 , erythromycin resistance was not detected [30]; since then, several pre-PCV studies have, however, shown the presence of erythromycin resistance of up to $28 \%$ in Ghana [15-17]. In this study (Table 3, Fig. 2), 16\% of erythromycin non-susceptible isolates were observed. Hence, it seems that erythromycin resistance has not changed greatly since the PCV-13 introduction.

Overall, we found that $20 \%$ of all the carriage isolates could be defined as MDR isolates, of which more than $60 \%$ of the serotypes were covered by the PCV-13 (Fig. 2). We furthermore observed a reduction of penicillin non-susceptible isolates covered by the PCV-13 compared to the study by Dayie et al. [13], and found that PCV-13 still covers most of the MDR isolates (Table 2). There is, therefore, still a possibility for great effect on reducing non-susceptible isolates with continuous $\mathrm{PCV}-13$ vaccination.

Ten isolates were found to be non-typeable serotypes, of which we were able to perform WGS on seven of the non-typeable isolates (Table 3). The seven NT isolates were differentiated into only two possible genotypes, genotypes 38 and 14 . One of the isolates, C140 with the ST 9735 showing a preferred genotype 14, also showed the same MLST type as ID 23690 (MLST database, https://pubmlst.org/). Isolate ID 23690 was a serotype 14 isolate submitted from the 2011 Ghana project [13]. This could support the relatedness of isolate C140 and isolate G7 to serotype 14 isolates, which have lost the ability to present the capsular gene for serotype 14 .

Five of the MDR isolates were analyzed by WGS (Table 3 ). Four of the isolates of serotype 19A were found to belong to ST320 (CC 320), which is a well-known penicillin resistant serotype 19A clone that has been observed all around the world and in particular after the PCV-7 introduction in USA [31, 32]. All four 19A isolates also showed an identical PBP profile of 13-11-16, which is related to a penicillin MIC of 4 according to Li et al. [23].

The limitation of this study is that we did not include children that were $<11$ month of age, who in other studies from Africa have been shown to have a high carriage rate $[10,29]$. The study focused on children from nurseries and kindergarten, which do not include children below one year of age. However, by choosing this group of children we were able to compare to some extent the carriage prevalence found in the pre-PCV-13 study by Dayie et al. [13], in which the study subjects were also children from nurseries and kindergarten in Accra. Although, it also has to be mentioned that the pre-PCV-13 study by Dayie et al. [13] was conducted from March - July 2011 and this study was conducted from September to December 2016, which means that seasonal variation could be a possible factor that might have influenced carriage prevalence between the two studies. Nonetheless, regardless of the possible seasonal variability, it is our assertion that comparing the children in this study with the pre-PCV-13 study [13] have made it possible to see whether there had been any changes in the pneumococcal serotype distribution five years post-PCV-13 vaccination in Accra, Ghana.

\section{Conclusions}

The introduction of PCV-13 in Ghana has reduced the carriage prevalence of serotypes covered by the PCV-13 although it has not removed them from the nasopharynx five years after the introduction of the vaccine. However, the PCV-13 vaccination covers majority of the non-antibiotic susceptible isolates. A further reduction of nonsusceptible pneumococcal isolates is therefore within likelihood. Measuring the effect of PCV-13 vaccination by continuous monitoring of the serotype distribution is important to evaluate the effectiveness of PCV-13. In addition, an evaluation of an alternative vaccination schedule from $3+0$ to $2+1$ needs to be considered to obtain the full effect of PCV-13 vaccination.

\section{Additional file}

Additional file 1: Table S1. The table presents the serotype distribution data from Dayie et al. [13] and the data from the present study, thereby making it possible to compare the data from the two carriage studies. (DOC $118 \mathrm{~kb})$

\section{Abbreviations}

CPS genes: Polysaccharide genes; ERY: Erythromycin; EUCAST: European Committee on Antimicrobial Susceptibility Testing; LEV: Levofloxacin; MDR: Multidrug-resistant; MIC: Minimum inhibitory concentrations; MLSA: Multilocus Sequence Analysis; MLST: Multilocus sequence typing; NT isolates: Non-typeable isolates; PBP: Penicillin-Binding Protein; PCV-10: 10- 
valent pneumococcal conjugate vaccine; PCV-13: 13-valent pneumococcal conjugate vaccine; PCV-7: 7-valent pneumococcal conjugate vaccine; PEN: Penicillin; ST: Sequence type; STGG: skim milk-tryptone-glucose-glycerin medium; SXT: Trimethoprim-sulphamethoxazole; TET: Tetracycline; WGS: Whole genome sequencing

\section{Acknowledgements}

We wish to thank the Ministry of Health and Education of Ghana as well as the parents of the study subjects for having given us the permission to carry out the research on their children.

We also wish to thank Torben and Alice Frimodts Foundation for their financial assistance given for the pneumococcal identification. We are sincerely grateful to Kirsten Burmeister and Monja Hammer for their skilled laboratory work and input to this study.

\section{Authors' contributions}

NTKDD conceived and designed the study. NTKDD, EYT, MJN, ESD, HCS contributed to the protocol writing. EYT collected the clinical samples. NTKDD, YET, MJN conducted the laboratory assays. NTKDD, EYT, MJN, ESD, HCS analyzed the data. NTKDD, EYT, ESD, HCS drafted the manuscript. NTKDD, EYT, MJN, EB, ESD, KAL, HCS reviewed the data and critically revised the manuscript. All authors have read and approved the final manuscript

\section{Funding}

The funding received from the Office of Research, Innovation and Development of the University of Ghana (Grant no. URF/9/ILG-068/2015-2016) for this study is gratefully acknowledged. We also wish to thank the Torben and Alice Frimodts Foundation for the financial assistance given for the

pneumococcal identification. The funding bodies did not have any role in study design, data collection, analysis, and interpretation of data, decision to publish, or preparation of the manuscript.

\section{Availability of data and materials}

The data and materials are available on request from the corresponding author (Hans-Christian Slotved, Ph.D., Senior Scientist, Department of Bacteria, Parasites and Fungi, Statens Serum Institut, Artillerivej 5, DK-2300 Copenhagen, Denmark, Tel: + 453268 8422, E-mail: hcs@ssi.dk.).

\section{Ethics approval and consent to participate}

Ethical approval for this study was obtained from the Ethics and Protocol Review Committee of the College of Health Sciences, University of Ghana (CHS-Et/M.9-P4.3/2015-2016).

Nasopharyngeal swab samples as well as demographic data were obtained from the participants after written consent had been obtained from the parents/guardians of the study subjects followed by a verbal consent from the children themselves (age $\leq 5$ years of age). If a child whose parents/ guardian had given written consent declined assent, the child was excluded from the study.

\section{Consent for publication}

Consent for publication does not apply.

\section{Competing interests}

Hans-Christian Slotved is involved with projects supported by Pfizer. All other authors had no conflicts of interest.

\section{Author details}

${ }^{1}$ Dept. of Medical Microbiology, School of Biomedical and Allied Health Sciences University of Ghana, Accra, Ghana. ${ }^{2}$ Department of Bacteria, Parasites and Fungi, Statens Serum Institut, Artillerivej 5, DK-2300 Copenhagen, Denmark.

Received: 13 March 2019 Accepted: 26 August 2019

\section{Published online: 05 September 2019}

\section{References}

1. Wahl B, O'Brien K, Greenbaum A, Liu L, Chu Y, Majumder A, et al. Global, regional, and national burden of Streptococcus pneumoniae and Haemophilus influenzae type b disease in children in the era of conjugate vaccines: updated estimates from 2000-2015. Lancet Glob Heal. 2018;6: e744-57. https://doi.org/10.1016/S2214-109X(18)30247-X.30.
2. Fjeldhøj S, Laursen RP, Larnkjær A, Mølgaard C, Fuursted K, Krogfelt KA, et al. Probiotics and carriage of Streptococcus pneumoniae serotypes in Danish children, a double-blind randomized controlled trial. Sci Rep. 2018;8:15258. https://doi.org/10.1038/s41598-018-33583-9.9.

3. Navne JE, Børresen ML, Slotved HC, Andersson M, Melbye M, Ladefoged K, et al. Nasopharyngeal bacterial carriage in young children in Greenland: a population at high risk of respiratory infections. Epidemiol Infect. 2016;144: 3226-36.18.

4. Kobayashi M, Conklin LM, Bigogo G, Jagero G, Hampton L, Fleming-Dutra $\mathrm{KE}$, et al. Pneumococcal carriage and antibiotic susceptibility patterns from two cross-sectional colonization surveys among children aged $<5$ years prior to the introduction of 10-valent pneumococcal conjugate vaccine Kenya, 2009-2010. BMC Infect Dis. 2017;17:25. https://doi.org/10.1186/s12 879-016-2103-0.12

5. Roca A, Bojang A, Bottomley C, Gladstone RA, Adetifa JU, Egere U, et al. Effect on nasopharyngeal pneumococcal carriage of replacing PCV7 with PCV13 in the expanded Programme of immunization in the Gambia. Vaccine. 2015;33:7144-51.20

6. International Vaccine Access Center (IVAC), Johns Hopkins Bloomberg School of Public Health. Vaccine Information Management System (VIMS) Global Vaccine Introduction Report, [Month Year]. http://www.jhsph.edu/ research/centers-and-institutes/ivac/vims/. Accessed 18 June 2019.

7. Van Buynder P, Booy R. Pneumococcal vaccination in older persons: where are we today? Pneumonia (Nathan Qld). 2018;10:1. https://doi.org/10.1186/ s41479-017-0045-y.28.

8. Slotved H, Dalby T, Hoffmann S. The effect of pneumococcal conjugate vaccines on the incidence of invasive pneumococcal disease caused by ten non-vaccine serotypes in Denmark. Vaccine. 2016;34:769-74. https://doi. org/10.1016/j.vaccine.2015.12.056.25.

9. Neves F, Cardoso N, Souza A, Snyder R, Marlow M, Pinto T, et al. Population structure of Streptococcus pneumoniae colonizing children before and after universal use of pneumococcal conjugate vaccines in Brazil: emergence and expansion of the MDR serotype 6C-CC386 lineage. J Antimicrob Chemother. 2018; February:1-7.19.

10. Usuf E, Bottomley C, Bojang E, Cox I, Bojang A, Gladstone R, et al. Persistence of nasopharyngeal pneumococcal vaccine serotypes and increase of non-vaccine serotypes among vaccinated infants and their mothers five years after PCV13 introduction in The Gambia. Clin Infect Dis. 2018; September. https://doi.org/10.1093/cid/ciy726.27.

11. Lindstrand A, Kalyango J, Alfvén T, Darenberg J, Kadobera D, Bwanga F, et al. Pneumococcal carriage in children under five years in Uganda-will present pneumococcal conjugate vaccines be appropriate? PLoS One. 2016; 11:1-15.15.

12. Mulholland K, Satzke C. Serotype replacement after pneumococcal vaccination. Lancet (London, England). 2012;379:1387; author reply 1388-9. https://doi.org/10.1016/S0140-6736(12)60588-1.17.

13. Dayie NTKD, Arhin RE, Newman MJ, Dalsgaard A, Bisgaard M, Frimodt-Møller $\mathrm{N}$, et al. Penicillin resistance and serotype distribution of Streptococcus pneumoniae in Ghanaian children less than six years of age. BMC Infect Dis. 2013;13(5).

14. Leimkugel J, Adams Forgor A, Gagneux S, Pflüger V, Flierl C, Awine E, et al. An outbreak of serotype 1 Streptococcus pneumoniae meningitis in northern Ghana with features that are characteristic of Neisseria meningitidis meningitis epidemics. J Infect Dis. 2005;192:192-9. https://doi.org/10.1 086/431151.13.

15. Donkor ES, Newman MJ, Oliver-Commey J, Bannerman E, Dayie NTKD, Badoe EV. Invasive disease and paediatric carriage of Streptococcus pneumoniae in Ghana. Scand J Infect Dis. 2010:42:254-9.7.

16. Mills RO, Twum-Danso K, Owusu-Agyei S, Donkor ES. Epidemiology of pneumococcal carriage in children under five years of age in Accra, Ghana. Infect Dis (London, England). 2015;47:326-31. https://doi.org/10.3109/ 00365548.2014.994185.16.

17. Dayie NTKD, Arhin RE, Newman MJ, Dalsgaard A, Bisgaard M, Frimodt-Møller $\mathrm{N}$, et al. Multidrug-resistant Streptococcus pneumoniae isolates from healthy Ghanaian preschool children. Microb Drug Resist. 2015;21:636-42. https:// doi.org/10.1089/mdr.2014.0314.6

18. Satzke C, Turner P, Virolainen-Julkunen A, Adrian PV, Antonio M, Hare KM, et al. Standard method for detecting upper respiratory carriage of Streptococcus pneumoniae: updated recommendations from the World Health Organization pneumococcal carriage working group. Vaccine. 2014; 32:165-79. https://doi.org/10.1016/j.vaccine.2013.08.062.21. 
19. Slotved HC, Facklam RR, Fuursted K. Assessment of a novel bile solubility test and MALDI-TOF for the differentiation of Streptococcus pneumoniae from other mitis group streptococci. Sci Rep. 2017;7:7167. https://doi.org/1 0.1038/s41598-017-07772-x.24.

20. Bishop CJ, Aanensen DM, Jordan GE, Kilian M, Hanage WP, Spratt BG. Assigning strains to bacterial species via the internet. BMC Biol. 2009;7(4).

21. Scholz CFP, Poulsen K, Kilian M. Novel molecular method for identification of Streptococcus pneumoniae applicable to clinical microbiology and 165 rRNA sequence-based microbiome studies. J Clin Microbiol. 2012;50:196873. https://doi.org/10.1128/JCM.00365-12.22.

22. Kapatai G, Sheppard CL, Al-Shahib A, Litt DJ, Underwood AP, Harrison TG, et al. Whole genome sequencing of Streptococcus pneumoniae: development, evaluation and verification of targets for serogroup and serotype prediction using an automated pipeline. PeerJ. 2016;4:e2477. https://doi.org/10.7717/ peerj.2477.11.

23. Li Y, Metcalf BJ, Chochua S, Li Z, Gertz RE, Walker H, et al. Penicillin-binding protein transpeptidase signatures for tracking and predicting $\beta$-lactam resistance levels in Streptococcus pneumoniae. MBio. 2016;7:1-9.14.

24. Zankari E, Hasman H, Cosentino S, Vestergaard M, Rasmussen S, Lund O, et al. Identification of acquired antimicrobial resistance genes. J Antimicrob Chemother. 2012;67:2640-4. https://doi.org/10.1093/jac/dks261.31.

25. Steens A, Caugant DA, Aaberge IS, Vestrheim DF. Decreased carriage and genetic shifts in the Streptococcus pneumoniae population after changing the seven-valent to the thirteen-valent pneumococcal vaccine in Norway. Pediatr Infect Dis J. 2015;34:875-83.26.

26. Sigurdsson S, Erlendsdóttir H, Quirk SJ, Kristjánsson J, Hauksson K, Andrésdóttir BDI, et al. Pneumococcal vaccination: direct and herd effect on carriage of vaccine types and antibiotic resistance in Icelandic children. Vaccine. 2017;35:5242-8. https://doi.org/10.1016/j.vaccine.2017.08.020.23.

27. van Hoek AJ, Sheppard CL, Andrews NJ, Waight PA, Slack MPE, Harrison TG, et al. Pneumococcal carriage in children and adults two years after introduction of the thirteen valent pneumococcal conjugate vaccine in England. Vaccine. 2014;32: 4349-55. https://doi.org/10.1016/.vaccine.2014.03.017.29.

28. Adetifa IMO, Adamu AL, Karani A, Waithaka M, Odeyemi KA, Okoromah CAN, et al. Nasopharyngeal pneumococcal carriage in Nigeria: a two-site, population-based survey. Sci Rep. 2018;8:3509. https://doi.org/10.1038/s415 98-018-21837-5.2.

29. Egere U, Townend J, Roca A, Akinsanya A, Bojang A, Nsekpong D, et al. Indirect effect of 7-valent pneumococcal conjugate vaccine on pneumococcal carriage in newborns in rural Gambia: a randomised controlled trial. PLoS One. 2012;7(8).

30. Holliman RE, Liddy H, Johnson JD, Adjei O. Epidemiology of invasive pneumococcal disease in Kumasi, Ghana. Trans R Soc Trop Med Hyg. 2007; 101:405-13.10

31. Beall BW, Gertz RE, Hulkower RL, Whitney CG, Moore MR, Brueggemann AB. Shifting genetic structure of invasive serotype 19A pneumococci in the United States. J Infect Dis. 2011;203:1360-8.3.

32. Žemličková $H$, Mališová $L$, Španělová $P$, Jakubů $V$, Kozáková J, Musilek $M$, et al. Molecular characterization of serogroup 19 Streptococcus pneumoniae in the Czech Republic in the post-vaccine era. J Med Microbiol. 2018::1-9. https://doi.org/10.1099/jmm.0.000765.

\section{Publisher's Note}

Springer Nature remains neutral with regard to jurisdictional claims in published maps and institutional affiliations.

\section{Ready to submit your research? Choose BMC and benefit from:}

- fast, convenient online submission

- thorough peer review by experienced researchers in your field

- rapid publication on acceptance

- support for research data, including large and complex data types

- gold Open Access which fosters wider collaboration and increased citations

- maximum visibility for your research: over $100 \mathrm{M}$ website views per year

At BMC, research is always in progress.

Learn more biomedcentral.com/submissions 